\title{
Charte de la Société Suisse de Médecine Interne pour la collaboration entre hôpital et cabinet médical
}

Les médecines hospitalière et ambulatoire forment une zone conflictuelle. Il en a toujours été ainsi, mais dans les conditions de développement tumultueux que l'on observe actuellement dans le système de santé publique, alors que des structures jadis solides se défont et que les démarcations claires s'estompent, ces conflits vont en augmentant. Il est en effet de plus en plus difficile de définir clairement et simplement où finit le domaine des uns et où commence celui des autres.

Entre les médecines hospitalière et ambulatoire il y a toute une série d'interfaces où les conflits et les problèmes les plus divers peuvent apparaître: sur le plan de l'organisation, des questions d'assurance, des compétences et des responsabilités, ainsi que sur le plan financier ou personnel.

Sur la base d'une large consultation effectuée auprès des collègues aussi bien en milieu hospitalier qu'ambulatoire, la Société Suisse de Médecine Interne a rédigé la présente charte dont l'objectif principal est d'inciter à aborder ouvertement les problèmes en suspens ou couvant sous la surface et d'en faciliter ainsi la discussion. En aucun cas le texte se veut une réglementation, et il n'a ni la prétention d'être exhaustif ni celle d'être applicable de manière générale. Mais dès le stade de son ébauche, ce document a déjà été utilisé comme base de discussion entre hôpitaux et praticiens, et il a été qualifié d'instrument utile.

De toute façon, une bonne collaboration ne peut s'établir que dans la mesure où l'on tient compte des conditions spécifiques données et que l'on recherche sans cesse le dialogue direct entre les médecins concernés.

Avec la charte pour la collaboration entre hôpital et cabinet médical, la SSMI espère pouvoir apporter une contribution à cette collaboration fructueuse.

W. Bauer, Président SSMI

\section{Charte SSMI pour la collaboration entre hôpital et cabinet médical}

\section{Données fondamentales}

- La collaboration et le partenariat entre les secteurs hospitalier et ambulatoire assurent à la population les meilleurs soins médicaux. A une époque où les structures interconnectées et les modèles de «disease management» se développent, les frontières entre les deux secteurs deviennent de plus en plus floues.

- Une coopération satisfaisante ne peut être obtenue que grâce à la volonté manifeste et l'engagement personnel des médecins concernés.

- Une collaboration fructueuse n'est possible que si les deux côtés recherchent activement la communication avec l'autre. Cela vaut aussi bien pour le praticien, dont les données et les informations récoltées doivent être transmises lors de l'hospitalisation d'un patient, que pour le médecin en milieu hospitalier qui doit informer le praticien à la fin de l'hospitalisation et lui faire part en temps voulu des faits importants survenus lors de celle-ci.
- Les aspects économiques et les problèmes de la qualité doivent être acceptés et discutés comme réalités incontournables.

- Il faut donner leur chance à des modèles nouveaux ou améliorés de collaboration ou d'intégration partielle entre l'activité hospitalière et ambulatoire. Il peut leur revenir un rôle de pionnier pour les développements futurs.

- Comme prestataires de service, l'hôpital et le cabinet médical se trouvent également en situation de concurrence dans certains domaines. Cela ne saurait être trop demander que d'exiger un minimum de fair-play. Les contrôles ambulatoires effectués automatiquement par les hôpitaux après la sortie des patients heurtent souvent la sensibilité des médecins traitants ayant ordonné l'hospitalisation; mais en même temps, ceux-ci attendent sans autre une disponibilité entière de la part de l'hôpital.

- Jusqu'à ce jour, on n'a encore peu tiré profit des possibilités offertes par des projets communs de recherche clinique, surtout dans 
les domaines de plus en plus importants des systèmes médicaux intégrés, du «disease management», de la recherche «outcome», de la «decision analysis», en tenant compte des données économiques.

\section{Communication}

- Communiquer, c'est échanger des informations. Le bulletin d'hospitalisation avec toutes les données nécessaires trouve son équivalence dans le bref communiqué immédiat de sortie, de transfert dans une autre institution ou de décès; suivi du bulletin de sortie détaillé avec toutes les informations nécessaires à la prise en charge du patient.

- Il faut périodiquement évaluer quels sont les attentes et les besoins réciproques. Par des échanges réguliers entre les médecins hospitaliers et les praticiens (ou leurs représentants délégués), on peut optimiser et actualiser la communication dans les deux sens. Ces échanges permettent également de faire personnellement connaissance, ce qui suffit parfois à éviter ou atténuer les points de friction.

- Connaître les prestations et la disponibilité du partenaire est une condition nécessaire à une collaboration optimale. Les médecins hospitaliers doivent savoir si un praticien donné est en mesure d'effectuer certains exa- mens et traitements post-hospitaliers de manière compétente (enlever les fils de suture, enlever un plâtre, faire des examens radiologiques, des ECG, des sonographies, des analyses en laboratoire, de la chimiothérapie, etc.) et quel est sa disponibilité.

- Les échanges de rapports écrits et oraux sont un facteur décisif. Il est souhaitable de pouvoir disposer d'un bref compte rendu au moment de la sortie d'un patient - par fax ou, si la confidentialité des données est garantie, par téléphone ou e-mail. Un rapport complet devrait être entre les mains du praticien dans les cinq à dix jours après la sortie du patient et contenir toutes les informations relatives à la suite du traitement, à un éventuel changement de thérapie, à une nouvelle thérapie ou à toute autre mesure importante à prendre.

- En faisant participer les praticiens au processus décisionnaire dans les questions touchant à des aspects médicaux et sociaux importants (placement en EMS, mesures de tutelle, interventions thérapeutiques ou diagnostiques lourdes chez des patients gravement malades ou âgés), on parvient à augmenter la qualité et l'acceptation de ces décisions auprès des patients et de leurs proches.

- Inversement, les médecins hospitaliers peuvent souhaiter disposer d'un rapport décrivant l'évolution de certains patients montrant une pathologie particulière.

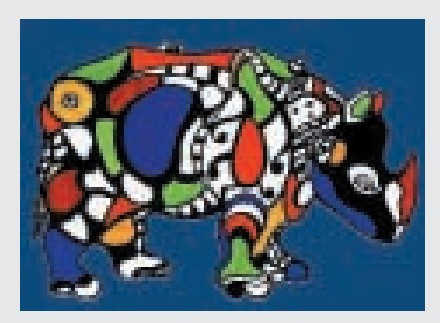

71 ${ }^{\text {e }}$ Assemblée annuelle

de la Société suisse de médecine interne

et le Congrès annuel

de la Société suisse d'hématologie

de l'Association professionnelle suisse de gériatrie

de la Section pharmacologie clinique et toxicologie

de la Société suisse d'hypertension

Lieu et date: Bâle, mercredi 21 mai au vendredi 23 mai 2003

Le track clinique: l'occasion d'apprendre non seulement les nouveautés mais les points cruciaux.

Clinical year in review: Des représentants de différentes disciplines médicales proposent un tour d'horizon sur les nouveautés scientifiques et d'intérêt clinique de leur discipline.

Clinical pearls: Les médecins-chefs de service de la Suisse du Nord-Ouest présentent des cas particuliers.

Workshops: Des médecins de premier recours et des experts étudient un thème en petits groupes avec le public.

Skill lab: informatique, mesure de la tension artérielle, gastroscopie, bronchoscopie,

techniques d'inhalation, toucher rectal, études autodidactiques en radiologie, ergonomie au travail.

Examen afin d'acquérir la reconnaissance en tant qu'expert en radioprotection de l'OFSP: 21 mai 2003, $11 \mathrm{~h} 45$.

Secrétariat administratif: Assemblée annuelle SSMI 2003, c/o AKM Congress Service, Case postale, 4005 Bâle, tél. 06168677 11, fax 06168677 88, e-mail: info@akm.ch

Site web avec des informations: www.akm.ch/sgim2003 
- Selon les possibilités et les besoins locaux, on peut envisager un échange d'informations et de pensées plus étroit: mise en place d'un système permettant de joindre directement les médecins cadre par téléphone, possibilités offertes par Internet, Hotline, conférences par téléphone, séances de discussion informelle entre médecins chefs de clinique, groupes de mise au point thérapeutique hôpital / cabinet médical, échange des listes de médicaments ou d'autres directives internes, participation à des visites médicales.

\section{Formation continue}

Bien qu'ils n'aient pas d'instructions clairement définies dans ce domaine, les services hospitaliers médicaux jouent un rôle essentiel dans la formation continue des jeunes médecins dans leur région.

- Les praticiens apprécient une formation continue qui leur permet d'élaborer librement et de manière modulaire leur propre programme en choisissant parmi les visites de congrès, les séjours dans des universités et des hôpitaux régionaux, la lecture et les médias numériques (qui prennent un place de plus en plus importante).

- Aujourd'hui, les formes interactives d'apprentissage et de transmission du savoir occupent le devant de la scène, mais cela ne veut pas dire que le cours magistral suivi de discussions libres n'aura plus sa place.

- De manière générale, il faut exiger que les cours de formation continue soient soumis à une évaluation.

- Quand cela est possible et utile, les médecins en formation continue aiment recevoir des notes écrites; selon les thèmes, il peut s'agir des instructions internes de l'hôpital qui peuvent se révéler fort précieuses pour les praticiens chargés du suivi post-hospitalier.
- Il faudra évaluer et tester sur place la possibilité de programmes d'échanges entre les hôpitaux et les cabinets médicaux, en y incluant les modèles d'assistanat en cabinet.

\section{Perspectives}

- L'idée selon laquelle tel type de patient ou de pathologie «revient ou appartient» automatiquement à un praticien, un groupe de spécialistes ou à une clinique est devenue de plus en plus indéfendable.

- La répartition rigide des tâches entre les divers acteurs du système de santé publique praticiens, spécialistes, hôpitaux, soins à domicile, centres, EMS, établissements de cure ou de rééducation et autres - s'estompe de plus en plus. La demande, la situation du marché et de concurrence, les développements techniques et structuraux, les aspects financiers et d'autres facteurs conduisent à une transformation des structures en place. Ce changement doit être mis à profit par la mise en place de structures interconnectées.

- A l'avenir, le contrôle et la gestion de la qualité dans les domaines du diagnostic, de la thérapie et du suivi continu des patients pour tout le processus diagnostique et thérapeutique, auront une importance croissante. S'ils veulent éviter que des contraintes leur soient imposées de l'extérieur, les praticiens et les hôpitaux doivent faire preuve d'initiative personnelle et prendre eux-mêmes les choses en main dans ce domaine.

- Les recommandations formulées dans cette charte valent également pour les relations entre les médecins exerçant dans de grands hôpitaux ou de futures organisations de la santé disposant d'un secteur hospitalier et d'un secteur ambulatoire (cas de figure: un hôpital qui comprend des cabinets médicaux ou un réseau de cabinets affiliés qui gère un hôpital). 